\title{
Los significados del deporte de competición para las mujeres deportistas
}

\section{The meanings of competitive sport for female athletes}

\author{
Belén Donoso Pérez \\ Universidad de Córdoba \\ belen.donoso@uco.es \\ https://orcid.org/0000-0002-4921-4283 \\ Alberto Álvarez-Sotomayor \\ Universidad de Córdoba \\ aasotomayor@uco.es \\ http://orcid.org/0000-0001-6174-1740 \\ Amalia Reina Giménez \\ Universidad de Córdoba \\ amalia.reina@uco.es \\ https://orcid.org/0000-0003-3916-9017
}

\section{Palabras clave}

- Deporte de competición

- Significados

- Identidad

- Género

- Investigación Cualitativa

- Teoría Fundamentada

\section{Resumen}

El estudio de los significados del deporte es una de las cuestiones más desarrolladas dentro de la sociología del deporte. No obstante, detectamos una escasez empírica de investigaciones que los aborden desde una perspectiva de género y fenomenológica. Este estudio analiza los significados que 45 mujeres deportistas le atribuyen a la práctica deportiva competitiva desde esta perspectiva. Se realizaron cinco grupos focales conformados en función del deporte practicado: futbol sala, deportes individuales (natación, atletismo y esgrima) de categoría senior, voleibol, balonmano y atletismo de categoría veterana. Los datos muestran una multiplicidad de significados. Las mujeres conciben el deporte como una forma de vida, como una herramienta para ordenarla, como fuente de sustento económico, como una vía de escape ante las obligaciones diarias, como un "flotador" al que agarrarse para sobrellevar las adversidades vitales, y como una forma de reivindicación de los derechos de las mujeres. Concluimos que el conocimiento de sus subjetividades en cuanto al lugar que le reservan al deporte en sus vidas y, con ello, de la importancia que adquiere en términos identitarios, constituye un elemento más para cambiar la perspectiva que adoptan las miradas y prácticas discriminatorias a las que se enfrentan en los escenarios deportivos. 


\section{Key words}

- competitive sports

- Meanings

- Identity

- Gender

- Qualitative Research

- Grounded Theory

\begin{abstract}
The study of sport's meanings is one of the most developed subjects within the sociology of sport. However, we have detected an empirical paucity of research that addresses them from a gender and phenomenological perspective. This study explores the meanings that 45 female athletes who live in Córdoba's province attribute to competitive sports practice from this perspective. Five focus groups were carried out, which were formed according to the type of sport practiced: indoor soccer, individual sports (swimming, athletics and fencing) of the senior category, volleyball, handball and veteran category athletics. The data produced show a multiplicity of meanings such as locating it as his way of life; the tool to organize it, the source of economic sustenance, an escape route from daily obligations, a "float" to hold on to cope with vital adversities; and a form of vindication of women's rights. The knowledge of their subjectivities in terms of the place they reserve for sport in their lives and, with this, the importance it acquires in terms of identity constitutes one more element to change the perspective adopted by the unequal views and practices that they face in sports venues.
\end{abstract}

\section{INTRODUCCIÓN}

La discusión sobre el significado del término deporte ha desencadenado (y continúa haciéndolo) cierta controversia debido a su extraordinaria polisemia, pudiendo incluir este desde el simple paseo, hasta la actividad profesional competitiva de alta competición. En este marco, y como resultado tanto del carácter abierto y cambiante del deporte (García Ferrando y Llopis 2017), como de las múltiples funciones que desempeña individual y socialmente, "la palabra deporte ha adquirido en nuestros días una proliferación de significados de tal magnitud que nos resulta difícil saber con claridad a qué hace referencia cuando alguien la emplea" (Moscoso 2006, 178).

Formalmente, y pese a la falta de consenso académico al respecto (García Ferrando y Llopis 2017), el deporte puede definirse como "toda forma de actividad física que, mediante la participación casual u organizada, tienda a expresar o mejorar la condición física y el bienestar mental, estableciendo relaciones sociales u obteniendo resultados en competición a cualquier nivel" (UNISPORT 1993, 5). Como se aprecia, esta definición incorpora una distinción tipológica fundamental, la cual, si bien con ciertos matices terminológicos, se establece como básica para los trabajos sociológicos que abordan esta cuestión (Alfaro 2008; García Ferrando y Llopis 2017; Moscoso,
Fernández y Rodríguez 2014; Vázquez 2002): aquella que separa el deporte recreativo o popular, del deporte de competición o de rendimiento. Se entiende por este último aquel que está encaminado a lograr altos niveles de ejecución para conseguir el éxito en la competición deportiva (Alfaro 2008). En él nos centraremos en este trabajo.

Pero más allá del entendimiento del significado del deporte -y, en particular, del de competición-a partir de definiciones formales y generalistas, resulta de sumo interés estudiar el deporte desde los significados que las propias personas le otorgan dentro de sus vidas. El conocimiento de estos significados ayuda a entender el sentido que le dan los individuos a la práctica deportiva, lo que contribuye a construir el deporte en sí mismo como hecho y fenómeno social. A este respecto, de las distintas formas que la sociología ha tenido de acercarse al estudio del deporte (García Ferrando 1990), el denominado enfoque fenomenológico resulta especialmente idóneo por centrarse precisamente en el estudio de esta materia a partir de las subjetividades sociales, en otras palabras, de aquellos rasgos que para las personas distinguen o caracterizan a este fenómeno social (Moscoso 2006).

Se parte entonces del reconocimiento de que estos significados se construyen a partir de la subjetividad social de los sujetos y, con ello, de que están 
marcados, entre otros factores, por los grupos sociales a los que estos pertenezcan y por la posición que ocupen en la estructura social. El género emerge entonces como una de las variables ineludibles. Dada su influencia en la estructura social del deporte (Sánchez, Moscoso y Piedra 2020) la perspectiva de género se torna en esencial para el análisis contemporáneo de este fenómeno (Moscoso 2008). Así se ha entendido, en general, desde la sociología del deporte y, más concretamente, desde el desarrollo de esta disciplina en España, tal y como evidencia el notable incremento de estudios sociológicos que analizan la situación de las mujeres en el deporte durante las últimas décadas en este país (Alfaro 2008, Piedra 2019; Puig y Soler 2004, Sánchez, Moscoso y Piedra 2020, Vázquez 2002).

Sin embargo, a pesar de, por un lado, este crecimiento de la perspectiva de género en los estudios sociológicos del deporte en España y, por otro lado, del hecho de que la cuestión del significado del deporte haya sido una de las áreas temáticas más desarrolladas por la sociología del deporte en este país (Moscoso 2006, 192), detectamos una llamativa escasez de investigaciones que, desde una perspectiva fenomenológica y, de manera más amplia, microsociológica, exploren los significados del deporte de competición para las personas que lo practican; más aún, cuando se trata de mujeres. ¿Cómo entienden y valoran las deportistas de competición el deporte? ¿Qué es para ellas? ¿Qué significado o significados tienen?

Con este artículo pretendemos contribuir a dar respuesta a estas preguntas. Es decir, desde una perspectiva fenomenológica y de género, este trabajo tiene como objetivo detectar y analizar los significados que las mujeres deportistas de competición le atribuyen a este tipo de deporte para de esta manera conocer mejor cómo el deporte está integrado en sus vidas y en sus identidades como mujeres.

\section{METODOLOGÍA}

Este trabajo parte de una investigación más amplia (Donoso, Reina y Álvarez-Sotomayor 2020; Donoso 2021) que tenía como principal objetivo conocer cómo se construye la identidad de las mujeres que practican deporte de competición y el papel que desempeña el deporte en este proceso desde una perspectiva psico-socio-cultural. La metodología es de carácter cualitativo. La muestra la componen 45 mujeres residentes en la provincia de Córdoba (España) que fueron seleccionadas con base a tres criterios de inclusión: ser mujer; ser mayor de 18 años; y, como criterio deportivo, haber participado en al menos un Campeonato de España en el caso de las deportistas individuales, o, en el caso de los deportes colectivos, que el equipo jugara en una División Nacional o estuviera aspirando al ascenso. Los deportes representados en este estudio son: el atletismo, la natación, la esgrima, el balonmano, el voleibol y el fútbol sala. Para la selección de las participantes se siguieron dos procedimientos diferentes de muestreo no aleatorio. Para los deportes colectivos, se contactó con los entrenadores de los equipos que respondían a los criterios de la investigación y que accedieron a participar. Para los individuales, se contactó con algunas deportistas conocidas por el equipo investigador, a partir de las cuales se completó el resto de la muestra mediante un muestreo de bola de nieve.

La técnica de investigación utilizada fue el grupo focal. Concretamente, se llevaron a cabo cinco grupos focales para cuya conformación se tuvieron en cuenta tres criterios: el deporte practicado, si el deporte era individual o colectivo, y la edad de las participantes. Así, tres de los grupos estuvieron compuestos por jugadoras de deportes de equipo: el primero por 9 jugadoras de fútbol sala, el segundo por 11 jugadoras de balonmano y el tercero por 10 jugadoras de voleibol. Los otros dos grupos focales fueron conformados con las deportistas individuales: uno con mujeres "jóvenes", con edades comprendidas entre los 20 y los 38 años practicantes de atletismo, natación y esgrima; y otro de mujeres practicantes de atletismo de categoría veterana, con edades a partir de 45 años. La inclusión de deportistas veteranas nos daba la posibilidad de detectar diferencias discursivas inter-generacionales en caso de que las hubiera.

Los grupos fueron conducidos por una investigadora que ejercía el rol de moderadora y, aunque su desarrollo se orientó inicialmente bajo un mismo guion inicial construido alrededor de los objetivos iniciales de la investigación, este se fue alterando y reconstruyendo por medio de las intervenciones e interacciones discursivas de las participantes. En el caso de los resultados aquí expuestos, el discurso analizado emergió, en gran medida, a partir de la pregunta inicial con la que se abrieron los grupos: "¿qué es para vosotras el deporte de competición?".

Aprovechando los rasgos que caracterizan a esta técnica (Krueger y Casey 2015; Morgan 1997), con los grupos se pretendió crear un espacio en el que las mujeres pudieran expresar libremente sus percepciones y experiencias. En este sentido, su diseño y desarrollo intentó facilitar una discursividad abierta y espontánea donde tuvieran cabida también las contradicciones y diferencias que pudieran existir entre las distintas participantes o entre las distintas posicio- 
nes de una misma. La duración de los grupos estuvo en todos los casos en torno a una hora y media. Todas las participantes se prestaron voluntarias a participar en ellos y firmaron un consentimiento informado.

Para el análisis de la información producida, y siguiendo la Teoría Fundamentada (Strauss y Corbin 2002), se llevó a cabo un proceso de codificación abierta en el que se identificó la categoría principal de análisis a la que se le asignó el código "Significados del deporte". La codificación abierta fue inductiva o emergente y se realizó a partir de las subjetividades de las participantes. De forma simultánea, en la codificación axial la categoría principal se fue construyendo y ligando de forma sistemática a las subcategorías hasta que se integraron, y finalmente formaron el esquema explicativo presentado en el apartado de resultados. La codificación axial alude "a la operación físico-manipulativa concreta por la que se asigna cada una de las unidades de significado a la categoría o categorías en la que se considera incluida" (Trigueros, Rivera y Rivera 2018, 24). En este caso, las unidades de significado se operativizaron en turnos y extractos discursivos. Los turnos son intervenciones individuales que tienen sentido en sí mismas. Los extractos discursivos son secuencias interactivas de varias participantes alrededor de un contenido analítico concreto e integrados por turnos.

Los códigos que se asignaron a cada una de las subcategorías fueron los siguientes: forma de vida, herramienta para ordenar la vida, vía de escape, fuente de sustento económico, flotador y forma de reivindicación por los derechos de las mujeres. En este caso particular de estudio, nos hemos decantado por el uso de códigos en vivo, que son los que se utilizan "cuando el nombre del código está obtenido directamente del lenguaje de los actores" (Andréu, García-Nieto y Pérez, 2007, 70). A cada participante se le asignó un seudónimo para garantizar su anonimato. Durante todo este proceso nos servimos del software NVivo 11 Plus.

\section{RESULTADOS Y DISCUSIÓN}

El análisis de la información producida a través de los grupos focales nos permitió encontrar que las participantes otorgan múltiples significados al deporte de competición. En concreto, distinguimos un total de seis, representados en el diagrama 1 en las seis subcategorías derivadas de la categoría analítica general. Como podrá verse, son, en la mayoría de los casos, significados que traslucen funciones que el deporte de competición cumple dentro de sus vidas de acuerdo con su propia percepción.
Diagrama 1.

Mapa de la categoría "Significados del deporte"

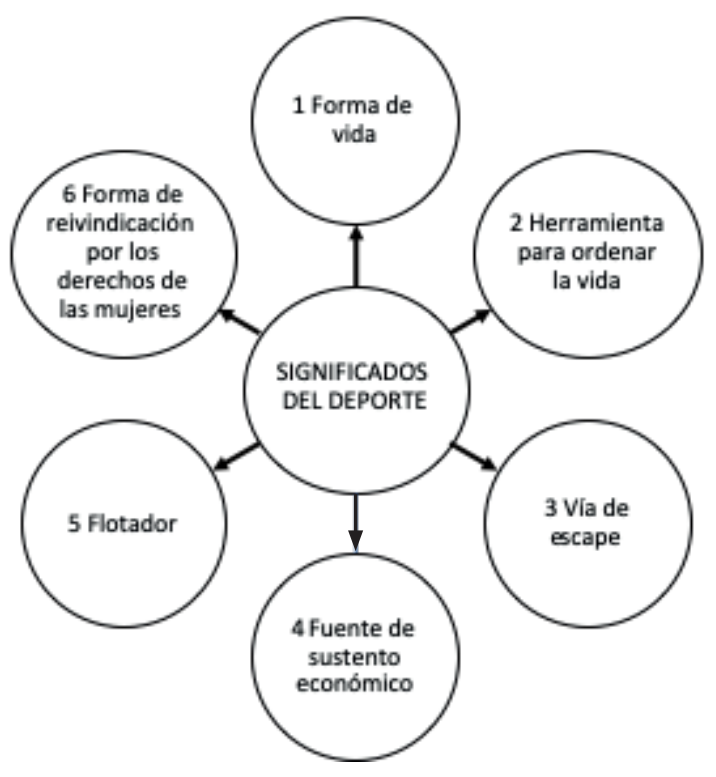

\section{El deporte como forma de vida}

En el discurso de las participantes, el deporte de competición se presenta, en primer lugar, como un elemento que define y estructura sus vidas. El deporte no es una actividad puntual cuya acción e influencia quede restringida al propio ámbito espacial, temporal y social en el que se desarrolla; el deporte es, para estas mujeres, una forma de vida. Este significado emerge como la categoría analítica con el mayor número de referencias codificadas y está presente en todos los grupos focales analizados, independientemente de la disciplina deportiva, de cómo esta sea etiquetada socialmente y de la edad de las participantes. Esto muestra el peso que tiene el deporte como eje vertebrador de sus vidas $y$, en consecuencia, como elemento configurador de sus identidades. Cada uno de los relatos que presentamos para ilustrar este hecho ofrece un matiz diferente que enriquece el mismo telón de fondo: el deporte define y estructura las formas y estilos de vida de estas mujeres. Pero, ¿de qué maneras lo hace? En primer lugar, identificamos que, al estar tan incrustado en sus experiencias vitales, al ser un hábito tan interiorizado, el deporte se torna en una necesidad; las participantes sienten la necesidad física y mental de entrenar. Sienten, pues, una fuerte dependencia del deporte. Lo vemos claramente cuando, por ejemplo, explican cómo afecta el hecho de no poder entrenar, a causa de las lesiones o por incompatibilidades con otros ámbitos, al resto de facetas de sus vidas y a su estado anímico: 
Lucía: y creo que lo va a seguir siendo de momento [hace alusión a los próximos dos o tres años].... [...], cuando llevo algún tiempo sin hacer nada [de deporte], al final necesito tanto física como mentalmente...practicarlo... [jugadora de voleibol]

Patricia: pues yo, por ejemplo, en mi caso, considero que el deporte es como una forma de vida, ¿no? Después de tantos años, yo, por ejemplo, personalmente llevo trece años practicando este deporte y al final pues se convierte en una forma de vida, ¿no? No ves más allá de..., no puedo, en mi vida no..., no hay día en el que no pueda haber balonmano, por ejemplo. [jugadora de balonmano]

Alejandra: [...], para mí el deporte es mi medio, porque también cuando estás lesionada hay veces que te necesitas desintoxicar, pero a la vez te contradices, porque luego no eres capaz de estar más de dos días seguidos descansando, porque eso nos pasa a todas... [atleta]

Clara: a mí me pasa, además, que cuando empecé la carrera tuve que dejarlo y fue ahí cuando me di cuenta de a lo que me quería dedicar, porque estuve un año sin poder entrenar y... Lo pasé fatal. [esgrimista]

Asimismo, las participantes expresan el elevado grado de implicación que el deporte de competición que conlleva en términos de constancia, esfuerzo y tiempo de dedicación. En este sentido, es un elemento revelador la influencia que el deporte ejerce en la planificación del resto de actividades de la vida de las deportistas, situándose como eje configurador de sus formas de vida:

Teresa: para mí, una forma de vida. Lleva muchos años integrado en mi vida y para mí una forma de vida, [...], vamos, no tiene otro calificativo. Simplemente lo tengo integrado en mi vida. Yo cada domingo o cada fin de semana, planifico mi semana en función de las horas que le voy a echar al deporte $y$, bueno, pues el lunes voy a hacer esto, el martes aquello y planifico un poco la semana, el fin de semana, ósea, que está integrado en mi vida, claro, [...], y marca un poco la dinámica de las demás actividades, sí. [nadadora]

Raquel: yo también lo definiría..., el balonmano..., el deporte, como una forma de vida, puesto que toda gira en torno al deporte, tu vida se organiza alrededor de practicar el deporte que..., en este caso, es el balonmano. [jugadora de balonmano]

Esta profunda vinculación que sienten con el deporte provoca que lo sitúen como la columna vertebral de sus vidas y que lo identifiquen como una "pasión". Cuando no pueden darle rienda suelta a esta "pasión" se sienten "completamente perdidas", ya que les resulta extremadamente difícil, por no decir inconcebible, entender su vida sin el deporte. Una de las atletas utiliza un símil que eleva esta consideración a un nivel incluso superior, comparando lo que para una compañera significa el deporte con el valor que para una persona pueda tener su religión. De nuevo, este símil nos sugiere que la deportista concibe el deporte como un elemento central en su identidad, como algo "intocable" y, a priori, difícil de abandonar. En su conjunto, este significado atribuido por las participantes al deporte nos recuerda a esa concepción "cagigaliana" del deporte como fenómeno que "Io inunda todo". En este marco, agradecen que su entorno próximo conozca esta relación y que cuando no pueden entrenar, justifiquen y disculpen su estado de ánimo:

Susana: es una pasión creo, es una forma de vida, es algo que condiciona el resto de tus facetas de tu vida. Y yo creo que aquí sentiréis igual que yo, que no entiendes tu vida sin el deporte, ya no en plan competición o alto rendimiento, porque vamos pasando por diferentes etapas y depende de la edad o circunstancias que te rodean, pero creo que sin deporte no funcionamos, y prueba de ello es cuando tenemos alguna lesión o lo que sea... [...] Yo creo que ese es el motivo por el cual nos venimos un poco abajo y nos sentimos completamente perdidas, porque no entendemos nuestra vida sin movernos..., [...], el círculo más próximo conoce tu día a día, ya lo ve como normal: "no, es que ella sin deporte no funciona, déjala...". [atleta]

Isabel: [...], me hubiese gustado que antes Alejandra dijese lo que realmente es el atletismo para ella, porque siempre que habla de atletismo, ella siempre dice "para mí el atletismo es mi religión, es decir, no me lo toques..., me da igual lo que digan...ósea, habladme de mil cosas, pero no me toquéis el atletismo, porque para mí el atletismo es mi religión, cada uno tiene la suya y para mí, la mía es esta". 
Entonces, me hubiera gustado que ella dijese eso, porque es su manera de expresarlo y yo creo que es muy, quizás muy gráfica, porque cuando a lo mejor te dicen "¿tú lo dejarías?", y dices, ¿cómo abandonas tu fe? [...] Pues eso es el deporte al final. [atleta]

Para estas mujeres, esta "pasión" por el deporte tiende a compensar los sacrificios asociados a los entrenamientos y competiciones, los cuales han estructurado y definido su día a día durante muchos años de su vida:

Gloria: yo creo que para mí el deporte, en mi persona, se define perfectamente como pasión, porque creo que sin eso nunca no llevaría tantos años compitiendo y sacrificándome tanto, tantos días, tantas semanas, tantos años sin parar... [jugadora de futbol sala]

En este marco, otros de los elementos que puede desencadenar que el deporte se convierta en su forma de vida reside en que las relaciones de amistad y de pareja que establecen, acontecen en el ámbito deportivo. También en este sentido, el deporte es para ellas un elemento que "marca" sus vidas. Asimismo, algunas reconocen que les ofrece la posibilidad de vivir una forma de vida completamente distinta en términos de viajar y conocer diferentes tipos de personas a la que, si no fuera por el deporte, quizás, no habrían tenido acceso:

Ana: [...], también quiero puntualizar que llega un punto en el que se convierte en tu vida porque tus amigas son del mismo círculo, incluso tu pareja es del mismo círculo y al final no sales de eso y, quieras o no, marca tu vida... [jugadora de balonmano]

Mar: a mí quizás el deporte ha sido, una forma de vida totalmente distinta. A mi el deporte me ha aportado conocer a gente de otras ciudades, conocer a mis amigas... [jugadora de voleibol]

En este punto resulta de interés destacar los resultados del grupo de veteranas. En contra de lo que quizás cabría esperar, en ellas identificamos que el paso del tiempo no ha supuesto (al menos en el momento de realización de este estudio) un obstáculo que desplace al deporte como el pilar de sus vidas. Teniendo en cuenta los sacrificios, el esfuerzo y la constancia que conlleva, más aún con la entrada en el mundo laboral o la llegada de las hijas/os, sería es- perable que el deporte constituyera una etapa en la vida de estas mujeres y que, superado o alcanzado cierto nivel, descasaran en cierto grado de él para entregarse a otros espacios más acordes con el discurso social legítimo atribuido a las mujeres. Por el contrario, el deporte se mantiene en ellas como elemento vertebrador de su identidad y "sobrevive" a los requerimientos sociales estipulados. Dos atletas veteranas comentan a este respecto:

Sandra: para mí el deporte en mi vida ha sido todo. Desde mi hobby en un principio, luego ha sido mi forma de vida; incluso mi trabajo lo debo al deporte, es decir, que para mí el deporte 10 ha sido todo, y ahora mismo, dándole su espacio a mi familia, a mi pareja y a mi trabajo, mi deporte es uno de los pilares fundamentales de mi vida, lo sigue siendo. [atleta veterana]

Claudia: para mí, comenzó como un hobby, pero cuando me quise dar cuenta era una forma de vida. Mi vida giraba en torno al deporte, si me iba de vacaciones era porque hacíamos una maratón, sí cogíamos algún fin de semana y nos escapábamos, era para hacer alguna competición... [atleta veterana]

\section{El deporte como herramienta para ordenar la vida}

En esta multiplicidad de significados, y partiendo de la percepción de que el deporte define y estructura sus formas de vida, las participantes lo conceptualizan como una herramienta que les ayuda en la organización, integración y optimización de las actividades diarias. El deporte les "incita" a la organización y aprovechamiento del tiempo disponible para estudiar, trabajar, etc., porque las deportistas son plenamente conscientes de que tienen que invertir un elevado número de horas en el entrenamiento. Resulta curioso cómo destacan la incapacidad que tienen de gestionar con eficacia el día que tienen de descanso y los sentimientos de irresponsabilidad asociados que tienen por este motivo. En este sentido, las jugadoras de balonmano comentan:

Julia: el deporte te ayuda a aprovechar el tiempo....

Yolanda: el deporte te incita a la organización.... Todas a coro: sí.

Yolanda: y tú disfrutas más de tu tiempo..., de que tienes que estar con tu pareja, con tus amigos, estudiar..., lo aprovechas más porque 
sabes que luego no es momento para ti...

Gema: no lo tienes...

Patricia: yo para mí, ha sido perfecta la explica-

ción, yo pienso igual...

Julia: te hace como..., te obliga...

Yolanda: es que te sale solo...

Julia: es que te hace seguir el ritmo porque a eso no puedes fallar...

Paola: la tarde libre al final no se hace nada... Yolanda: ¿la tarde libre...?

Julia: la tarde libre no hago nada..., [risas]

Paola: ni se mueve, ni se mueve...

Julia: pues yo el lunes no hago una puta mier-

da..., porque la siesta al final la haces hasta las tantas...

Patricia: hasta las 20:00 [risas].

Julia: te levantas... [risas]

Yolanda: comes como un cochinillo... [risas], es verdad, comemos demasiado... ¿estudiar?, no estudiamos, la siesta de tres horas...

Patricia: hasta las 20:00 como yo tío...

Bea: que te levantas hecha polvo...

Julia: y luego tienes que recuperarte de la siesta que te has echado..., y ya te da la hora de cenar y dices: "pues ya voy a cenar" ...

Paola: y me acuesto otra vez...

Yolanda: yo me siento hasta irresponsable...

Paola: Ios lunes que hay descanso, yo tengo esa sensación...

Yolanda: yo no soy yo, los lunes es como...no

Este último relato de Yolanda, en el que afirma no reconocerse a sí misma los lunes, el día que tiene de descanso, evidencia el papel del deporte como el elemento vertebrador de su identidad. El siguiente, de dos jugadoras de futbol sala, se sitúa en la misma línea, pero apuntando, en este caso, a la función liberadora que tiene el deporte y a la utilidad de esta en términos de aprovechamiento del tiempo; apuntando a cómo las horas que invierten entrenando les sirven para optimizar el estudio posterior gracias a la desconexión mental que esta actividad les proporciona:

Aurora: yo por ejemplo, en épocas de exámenes que te agobias mucho y dices, si no entrenara hoy, que tengo mucho que estudiar y tal [hace entrever que le vendría mejor], pero luego la hora que vienes a entrenar o las dos horas que vienes, llega otra vez y es como uf, me ha servido de desconexión y ahora empiezo otra vez y ¿sabes qué?, que parece que es pérdida de tiempo y luego te sale más rentable a la hora de... te sabes organizar mejor de cara a si tengo que ir a las ocho, pues hasta las ocho estudio tanto y claro si no tienes deporte pues a lo mejor no te organizas en torno a nada. [jugadora de futbol sala]

Daniela: te cuesta menos, aunque sea ponerte tarde, pero te cuesta menos ponerte esas dos horas que estar toda la tarde sin haber hecho el deporte. [jugadora de futbol sala]

Nuestros resultados, en la línea de otros previos (Gallego 2008; Kauer y Krane 2006), sugieren que las deportistas tienen en común una gran disciplina en la organización de los diferentes tiempos que dedican a cada una de sus actividades. La planificación y la organización se hacen indispensables para las mujeres deportistas, que tienen que compaginar un gran número de actividades y roles (estudios, trabajo o responsabilidades familiares) con la práctica deportiva (Gallego 2008). En este sentido, para muchas parece que el deporte les ha enseñado el valioso significado de la organización (Roessler y Muller 2018).

\section{El deporte como vía de escape}

El deporte, tal y como se ha visto, estructura y define la forma de vida de las deportistas y, salvo en los seis casos identificados en este estudio que pudieron dedicarse al deporte de forma profesional durante un periodo de sus vidas, el resto de las deportistas analizadas, a pesar de haber alcanzado un elevado nivel deportivo al competir a nivel nacional en ligas de primer nivel nacional (caso de los deportes colectivos) o en campeonatos de España con las marcas mínimas exigidas que permiten su asistencia (caso de las deportistas individuales), han tenido que conciliar este nivel de práctica competitiva con actividades de otra índole. Resulta significativo encontrar cómo, pese a todos estos sacrificios que el deporte conlleva, otro de los significados que le atribuyen nuestras participantes es el de ser una vía de escape que les permite desconectar de las obligaciones de la vida diaria y liberar las tensiones acumuladas.

En cuatro de los cinco grupos focales analizados encontramos referencias que contribuyen a reforzar este significado del deporte, independientemente de su etiquetado social y del rango de edad de las deportistas. En primer lugar, las características que principalmente transmiten para identificarlo como una vía de escape son, por una parte, la utilidad que le otorgan para liberar energía debido al alto nivel de activación que tienen y, por otra, conceptualizarlo como el momento que dedican exclusivamente a ellas mismas al margen de las obligaciones de la vida 
diaria, como elemento que les permite desconectar mentalmente de la cotidianeidad. El deporte, en este sentido, constituiría una herramienta de autocuidado para la mujer:

María Luisa: para mí es una forma de descargar adrenalina, yo es que lo necesito, tengo mucha energía y entre muchas actividades que hago al cabo del día, una de ellas es el deporte... [atleta veterana]

Araceli: [...], es para mí un pilar muy importante, sin él no creo que mi vida fuera igual. No sé, he sacrificado muchas cosas también y la verdad es que me da..., luego a la hora de practicarlo, me da muy buenas sensaciones, quemo mucha adrenalina con el deporte, me gusta..., no sé, coger una velocidad, una agilidad que me gusta, no sé, me da muy buena satisfacción... [...], me hace sentirme bien y por supuesto me cambia..., siempre lo practico por la mañana muy temprano y es otra forma de empezar el día, ¿no? Yo tengo mucha energía, soy una persona muy activa y entonces..., me tranquiliza... [atleta veterana]

Elena: yo mira, yo para mí es mi momento, es el que me dedico a mí, es..., no sé, una vía de escape, de hacerme un paréntesis en el día y decir: "ahora me toca a mi" y dejar todo lo que conlleva el día a día..., [...], ya te digo..., además de valorarme, pues es una vía de escape... [atleta veterana]

Claudia: [...], luego también..., te das cuenta también de que ya no solamente tu forma de vida, sino la manera que tú utilizas para liberarte, liberarte del estrés, liberarte de los problemas, en ese momento, como decía ella, eres tú, lo haces para ti y te liberas, y te liberas de muchísimas cosas, y eso pues te sirve. [atleta veterana]

\section{El deporte como fuente de sustento económico}

En los mejores casos, las deportistas, por un complejo conjunto de razones tanto individuales como contextuales o circunstanciales, consiguen alcanzar un elevado rendimiento que les permite poder utilizar el deporte como única forma de sustento económico. Esta situación desencadena que le atribuyan un significado asociado a una utilidad económica: el deporte como herramienta para ganarse la vida. Para ilustrar esta situación, exponemos, en primer lugar, los relatos de dos jugadoras de balonmano procedentes de Chile y Rumanía para las que el deporte ha supuesto una vía para la movilidad social ascendente. El deporte se presenta en este marco como una oportunidad para ambas jugadoras de salir de sus respectivos países y poder vivir de él. En este sentido, se convierte en un recurso clave para las familias con escasos recursos y/o en países en vías de desarrollo (Galatti et al. 2019). Estas dos jugadoras apuntan a este respecto:

Paola: yo lo veo como una oportunidad. Nunca pensé en ser deportista ni nada, solo que me gustaba mucho el deporte y estoy aquí, $y$ antes hice muchas cosas y hasta que me convencieron para el balonmano, entonces me vine, seguí entrenando, vinieron los torneos, después me vine aquí a la liga y me cogieron..., después, así como nada planeado, ni nada como forma de vida, sino como una oportunidad que.... y bueno, [...], también con sus altibajos, con el esfuerzo y el sacrificio que uno tiene que tener para ser deportista, pero como una oportunidad, y no solo para mí, sino creo que para gente en riesgo social o para todo el mundo, la oportunidad de estar aquí. Yo, por ejemplo, yo estudié en Chile, y yo nunca en mi vida decía, yo...., mi sueño es vivir en España, o vivir por aquí, no..., hasta que se dio..., así como eso..., a la gente le puede venir como un círculo, entonces creo que es como una oportunidad...

Sofía: yo pienso igual que ella, porque yo cuando empecé igual, yo practiqué diferentes deportes, y para mí cuando empecé..., ahora lo pienso y me hace gracia, teníamos un grupo de amigas y pues lo típico en mi país por esa época no había tanta libertad como ahora de salir a la calle y yo lo usaba en plan tengo que entrenar..., [...], luego al tiempo empecé pues a comprometerme seriamente con ellos, empezó a gustarme y ya..., mis padres también me apoyaron y empecé a dedicarle el tiempo que requería y siempre he sido comprometida pero sí es una vía de..., una oportunidad para conocer diferentes tipos de personas, para moverte en diferentes sitios, conocer, viajar..., yo que sé, a mí me ha aportado muchísimas cosas y no sé, creo que nunca me voy a arrepentir de ello. [jugadora de balonmano]

En esta línea de resultados, una atleta y una nadadora que también pudieron utilizar el deporte como sustento económico en un momento determinado de 
sus vidas aclaran, no obstante, que no le atribuyen a este hecho un papel principal, sino que le añaden otros significados analizados con anterioridad, tales como identificarlo como su forma de vida y su importancia como elemento que optimiza la organización del día a día. Esta intervención encarna precisamente lo que evidencian nuestros resultados, que el deporte es para estas deportistas polisémico, y que tiene unas raíces profundas arraigadas en su identidad:

Raquel: para mí es una forma de vida, para mí ha sido personalmente una forma de vida, una forma de ganarme también la vida, con algo que me apasiona. [atleta]

Mercedes: yo coincido también con Raquel que, aparte de ser una forma de vida, una cosa que te estructura el día a día, una manera un poco de..., para mí era como estoy en el alto nivel, pero me puedo mantener en el alto nivel porque esto me está dando ahora mismo para mantenerme, me está dando dinero para mantenerme al día a día... [nadadora]

\section{El deporte como "flotador"}

La metáfora que encabeza este apartado, tomada de las palabras de una de las participantes, ilustra cómo el deporte puede ser concebido como un elemento de apoyo psicológico y emocional, como un sostén que en periodos difíciles de la vida ayuda a mantenerse a salvo, a flote. El deporte, en esos momentos, puede constituirse como un "refugio" en el que encontrar el consuelo y la fuerza necesaria para superar los obstáculos que la vida pone por delante. Nuevamente, el deporte vuelve a presentarse como una herramienta de autocuidado y protección, características que consideramos refuerzan la influencia de este en la configuración de la identidad de estas mujeres. Para ilustrar este significado, exponemos un extracto discursivo muy íntimo recogido en el grupo focal de las atletas veteranas. Durante su transcurso, varias de las participantes reconocieron que el deporte había sido una pieza clave en el proceso de superación de sus divorcios:

Ma José: para mí el deporte es, no sé..., es mi flotador..., mi..., digamos, mi mejor amigo ... No quiero que me llegue a estresar ni quiero pasarlo mal porque tenga que entrenar antes que comer, antes que salir, pero yo sé que cuando estoy mal, yo me refugio mucho más en el deporte..., y salgo, salgo para delante..., [...], porque es una cosa que está ahí siempre, el deporte desde hace 20 años siempre está ahí y cuando lo necesito siempre está ahí, el deporte y la gente que practica ese deporte... [atleta veterana]

Sandra: el salvavidas. [atleta veterana]

Araceli: quiero decir que llevo separada muy poco, seis meses, de hecho, todavía no está legalizado, y sin el deporte no podía haber sacado..., [...], que esto me ha ayudado mucho a..., vamos es que me da igual ya todo, que veo ya a la otra persona y digo: "pfff, madre mía..." [risas] [atleta veterana]

Sandra: que voy a correr más todavía... [atleta veterana]

Araceli: sí, sí, que me ha ayudado mucho...totalmente. [atleta veterana]

\section{Forma de reivindicación por los derechos de las mujeres}

Este particular significado del deporte ejemplifica el poder de reivindicar el derecho de las mujeres a practicar deporte y a situarlo en el centro de sus vidas. El telón de fondo que subyace reside en la culpabilidad generalizada que suelen sentir las deportistas que son madres por compatibilizar ambos roles y soportar la presión social cuando, según las expectativas sociales, descuidan el rol maternal. En este sentido, estas mujeres se alejan del discurso legítimo de "madre cuidadora a tiempo completo y en exclusividad" para incurrir en un terreno vedado para ellas y anteponerlo a las obligaciones sociales prescritas. En el relato que se recoge a continuación la atleta revive el momento en el que ella, abrumada por el cuidado de los hijos, con su marido lejos de casa y estudiando unas oposiciones, decide escucharse y ponerse a ella misma en el centro de su vida, aceptando la oportunidad de subir el Aconcagua. La atleta, que por obligaciones familiares y profesionales había desplazado uno de los pilares más importantes de su vida, siente que necesita volver a entrenar y plantearse un reto para superarse a sí misma. En su narración podemos observar las contradicciones vividas y el momento de detenerse y preguntarse a sí misma: "¿por qué los hombres no se plantean el no irse y yo por ser una mujer sí?", así como las presiones recibidas desde su entorno, que entiende que no se hubiesen dado si ella hubiera sido un hombre:

María Luisa: [...], yo con 40 años y dos hijos [...], mi marido estaba en Sevilla y yo estaba 
todo el día con ellos, y llegó un momento en que dije..., bueno, me salió una oferta: "¿quieres ir al Aconcagua?". Y dije: "¿y por qué no?, ¿y por qué no voy a hacerlo? Ahora me voy a dedicar a mí. Y pedí un año sabático, se puede decir .. a mi marido, a mis niños, les dije: "un año me voy a dedicar a mí, me voy a dedicar a la montaña". Me iba todos los fines de semana a Sierra Nevada, me fui al Kilimanjaro, Io hice todo en un año que tuve para mí, en un año a tope y era como una..., aparte de que fue..., era una cosa que, como mujer, reivindicar el deporte como mujer porque era un proyecto de decir: "Ios hombres se van a la montaña, ipor qué una mujer no puede hacerlo"? En Córdoba no ha habido ninguna que lo haga, tú tienes la oportunidad de ser la primera y lo vas a ser. Y entonces, yo lo hice, a pesar de que lo tenía todo en contra: la familia, los amigos..., todo en contra porque: "¿qué necesidad tienes?, dejar dos hijos, irte a una montaña, el peligro, tal y cual". Y lo hice porque yo podía, porque yo quería y quería hacerlo y me había sacrificado toda la vida, no me había sacrificado, pero yo no había leído la tesis porque tuve los hijos y dije: "no quiero hacer esto, quiero hacer lo otro". Había montado mi vida diciendo no, y llegó un momento en que dije: "ahora voy a hacer lo que yo quiero, sin importarme nada ni nadie", y 10 hice, y tiré para delante, un año con todas las oposiciones y tal..., [...] [atleta veterana]

Este relato sugiere que cuando la práctica deportiva la realizan las dos personas de una pareja heterosexual, a la mujer se le suman más dificultades, al tener que conjugar a menudo el rol tradicional de género (doméstico, reproductivo, y de crianza y cuidado) con el deportivo. Esto está en línea con los resultados de trabajos previos. Así, por ejemplo, Moscoso (2008) destaca cómo la "obligación" de las mujeres de hacer frente a estos roles dificulta la práctica del montañismo o, al menos, la ambición de conseguir retos deportivos más altos.

Nos parece oportuno destacar la posición que adoptaron las instituciones implicadas en el desarrollo del proyecto que narra la anterior atleta. Estas rechazaron financiar el proyecto por considerarlo irrelevante en términos de ayuda a las mujeres, al tratarse de un proyecto deportivo. Sin embargo, tras su consecución evidenciaron la importancia de visibilizar y apoyar a mujeres en ámbitos en los que pueden convertirse en referentes y generar, de esta manera, un motor de cambio y liberación en las generaciones futuras. A este respecto, la atleta comenta:
María Luisa: pero fue como una reivindicación..., a nosotras nos dieron el premio Meridiana, que yo no quería cogerlo, pero nos 10 dieron. No quería cogerlo porque tuve muchas críticas del Instituto Andaluz de la Mujer, de pedir dinero para una cosa que era "lúdica" ... Después, cuando la hicimos, vieron la importancia de que las mujeres hicieran cosas y que no solo era el dinero para las maltratadas, que también había que apoyar a las mujeres en otros ámbitos..., [...], y nos dieron el premio y yo les dije: "pues no lo quiero, porque yo he venido aquí a pedir vuestra ayuda y me dijisteis que mientras que hubiera una mujer maltratada, no gastamos el dinero en tonterías..." [atleta veterana]

\section{CONCLUSIONES}

El discurso de las participantes refleja el carácter polisémico que tiene el deporte para ellas y, con ello, la multiplicidad formas en las que se integra dentro de sus vidas y acaba contribuyendo a la construcción de sus identidades.

Tal y como se ha explicado, por encima de todo, las participantes identifican el deporte como una forma de vida. Esta atribución implica que el deporte se presenta como el eje que vertebra sus vidas y sobre el que organizan y planifican el resto de los ámbitos vitales. Del mismo modo, destacamos esa "necesidad" que tienen de practicarlo y cómo afecta el no poder hacerlo en su estado de ánimo y en el consiguiente sentimiento de ubicación en el mundo. El deporte se presenta también como un factor que optimiza la organización y aprovechamiento del tiempo en el resto de actividades vitales, como un elemento de sustento económico en los casos en los que las deportistas alcanzan el máximo nivel, y como un "flotador" en el que apoyarse para afrontar las adversidades.

El deporte es también percibido como una vía de escape, en tanto en cuanto les supone una forma de desconexión de las obligaciones y rutinas diarias. En este sentido, el deporte constituye un "refugio" en el que poder dedicarse a ellas mismas, se convierte en una herramienta de autocuidado para la mujer. Por último, el deporte supone en algunos casos una forma de reivindicación de sus derechos como mujeres, al convertirse en un espacio en el que reclamar la igualdad de género. La igualdad, en este caso, para practicarlo y para afrontar retos en las mismas condiciones que los hombres sin tener que verse sometidas a las presiones sociales por descuidar otros roles que continúan inequitativamente asociadas a ellas. 
En un espacio como el del deporte de competición, tan fuerte e históricamente marcado por las desigualdades de género (Fausto-Sterling 2006; Hargreaves 1994; Martín, Soler y Vilanova 2017), resulta revelador los significados que las participantes atribuyen al deporte competitivo, la gran importancia que le otorgan a esta práctica; la centralidad y expansión que tiene el deporte en las distintas facetas y ámbitos de sus vidas. Ello contrasta con las persistentes miradas y prácticas discriminatorias con las que otros agentes - tanto desde dentro (federaciones, entidades deportivas locales, medios de comunicación, etc.), como desde fuera de este espacio (entorno social de las deportistas, opinión pública, etc.)se acercan al deporte de competición practicado por mujeres, como revelan los trabajos que analizan dichas miradas y prácticas (Alfaro, Bengoechea y Vázquez 2010; Martínez-Abajo, Vizcarra y Lasarte 2020; Salido y Muñoz 2021) y aquellos que se centran en las percepciones que las propias deportistas tienen al respecto (Donoso 2021).

En este sentido, consideramos que la puesta en conocimiento de las subjetividades de estas mujeres en cuanto al espacio y el lugar que le reservan al deporte en sus vidas y, con ello, de la importancia que tiene este para ellas en términos identitarios, constituye un elemento más para cambiar la perspectiva que adoptan las miradas y prácticas discriminatorias mencionadas. De ahí la pertinencia social de este tipo de estudios. En lo académico, consideramos que este estudio debe constituir un punto de partida de otros a mayor escala que abarquen a un mayor número de participantes e incluyan a otras modalidades deportivas etiquetadas socialmente como femeninas, ausentes en esta investigación. En este sentido, se profundizaría en el conocimiento sobre los significados que las mujeres le atribuyen a la práctica deportiva competitiva desde una perspectiva fenomenológica y contribuiría a enriquecer esta área temática.

\section{REFERENCIAS}

Andréu, Jaime., García-Nieto, Antonio y Pérez, Ana. 2007. Evolución de la teoría fundamentada como técnica de análisis cualitativo. Madrid: Centro de Investigaciones Sociológicas.

Alfaro, Élida. 2008. Mujer joven y deporte. Revista de estudios de la juventud, 83: 119-141. http://www. injuve.es/sites/default/files/RJ83-09.pdf

Alfaro, Élida., Bengoechea, Mercedes. y Vázquez, Benilde. 2010. Hablamos de deporte: en femenino y en masculino. Madrid: Instituto de la Mujer (Ministerio de Sanidad, Política Social e Igualdad).
Donoso, Belén. 2021. La identidad de la mujery el deporte de competición desde una perspectiva psico-socio-cultural. (Tesis Doctoral). Universidad de Córdoba. https://helvia.uco.es/xmlui/handle/10396/21432.

Donoso, Belén. 2021. La identidad de la mujery el deporte de competición desde una perspectiva psico-socio-cultural. (Tesis Doctoral). Universidad de Córdoba. https://helvia.uco.es/xmlui/handle/10396/21432.

Fausto-Sterling, Anne. 2006. Cuerpos sexuados: la política de género y la construcción de la sexualidad. Tenerife: Melusina.

Galatti, Larissa R., Marques, Renato F., Barros, CarIos E., Montero, Antonio y Rodrigues, Roberto. 2019. Excellence in Women Basketball: Sport Career Development of World Champions and Olympic Medalists Brazilian Athletes. Revista de Psicología del Deporte 28(1): 17-23. https://ddd.uab.cat/record/219106

Gallego, Beatriz. 2008. La investigación biográfico-narrativa en un estudio sobre la situación de las mujeres en el deporte. Revista de Investigación Educativa, 26(1): 121-140. https://revistas.um.es/rie/article/view/94151/90771

García Ferrando, Manuel. 1990. Aspectos sociales del deporte. Una reflexión sociológica. Madrid: Consejo Superior de Deportes y Alianza Editorial.

García Ferrando, Manuel y Llopis, Ramón. 2017. "Estructura social de la práctica deportiva". En Sociología del deporte, editado por Manuel García Ferrando, Núria Puig, Francisco Lagardera, Ramón Llopis y Anna Vilanova, 39-68. Madrid: Alianza Editorial.

Hargreaves, Jennifer. 1994. Sporting Females. London: Routledge.

Kauer, Kerrie J. y Krane, Vikki. 2006. "Scary Dykes" and "Feminine Queens": Stereotypes and Female Collegiate Athletes. Women in Sport and Physical Activity Journal, 15(1): 42-55. https://doi.org/10.1123/wspaj.15.1.42

Krueger, Richard A. y Casey, Mary A. 2015. Focus groups. A practical guide for applied research. London: Sage.

Martín, Montse., Soler, Susana y Vilanova, Anna. 2017. "Género y deporte". En Sociología del deporte, editado por Manuel García Ferrando, Núria Puig, Francisco Lagardera, Ramón Llopis y Anna Vilanova, 97-123. Madrid: Alianza Editorial.

Martínez-Abajo, Judit., Vizcarra, María Teresa y Lasarte, Gema. 2020. How do Sportswomen Perceive the Way they are Treated in the Media?. Apunts. Educación Física y Deportes, 139: 73-82. https://doi. org/10.5672/apunts.2014-0983.es.(2020/1).139.10

Morgan, David L. 1997. Focus groups as qualitative research. London: Sage.

Moscoso, David. 2006. La sociología del deporte en España. Estado de la cuestión. Revista inter- 
nacional de sociología, 64(44): 177-204. https://doi. org/10.3989/ris.2006.i44.33

Moscoso, David. 2008. The social construction of gender identity amongst mountaineers. European Journal for Sport and Society, 5(2): 187-194. https:// doi.org/10.1080/16138171.2008.11687819

Moscoso, David, Fernández, Jesús y Rodríguez, Álvaro. 2014. De la democratización del deporte a la hegemonía de los mercados: el caso español. Movimento, 20: 109-124. https://www.redalyc.org/ pdf/1153/115335321010.pdf

Piedra, Joaquín. 2019. La perspectiva de género en sociología del deporte en España: presente y futuro. Revista Española de Sociología, 28 (3): 489-500. http://dx.doi.org/10.22325/fes/res.2019.13)

Puig, Núria y Soler, Susanna. 2004. Mujer y deporte en España: estado de la cuestión y propuesta interpretativa. Apunts. Educación física y deportes, 2(76): 71-78.

Roessler, Kirsten. K. y Muller, Ashley. E. 2018. "I don't need a flat tummy; I just want to run fast" - self-understanding and bodily identity of women in competitive and recreational sports. BMC Women's Health, 18, 146. https://doi.org/10.1186/s12905-018-0639-4
Salido, Juana y Muñoz, Ana. 2021. Representación mediática de las deportistas en los Juegos Olímpicos: una revisión sistemática. Apunts, Educación Física y Deportes, 146: 32-41. https://doi.org/10.5672/ apunts.2014-0983.es.(2021/4).146.04

Sánchez, Raúl., Moscoso, David y Piedra, Joaquín. 2020. "The sociology of sport in Spain: Development, current situation, and future challenges". Sport und Gesellschaft, 17(1): 69-95.

Strauss, Anselm y Corbin, Juliet. 2002. Bases de la investigación cualitativa: técnicas y procedimientos para desarrollar la teoría fundamentada. Antioquía: Editorial Universidad de Antioquía.

Trigueros, Carmen., Rivera, Enrique y Rivera, Irene. 2018. Técnicas conversacionales y narrativas Investigación Cualitativa con Software NVivo. Granada: Universidad de Granada y Escuela Andaluza de Salud Pública.

UNISPORT. 1993. Carta Europea del Deporte, Málaga, UNISPORT, Junta de Andalucía, Málaga.

Vázquez, Benilde. 2002. La mujer en ámbitos competitivos: el ámbito deportivo. FAISCA. Revista de Altas Capacidades, 9: 56-69. https://dialnet.unirioja.es/ servlet/articulo? codigo $=2476360$ 